\title{
The acid/base surface characterization of sandstone, limestone and marble and its effect upon the polymerisation of tetra- ethoxysilane
}

\author{
Elizabeth S. Goins \\ Institute of Archaeology, UCL
}

\section{Introduction}

The conservation of historically important stone buildings and monuments is a complicated field that crosses the boundaries of several different disciplines; in particular chemistry, geology, biology and materials science. It is a fact of nature that materials decay, and the role of the conservator is to delay this process for as long as possible. One way of approaching the problem is to place the object in a controlled environment. This is, however, clearly impossible in the case of large objects, and is expensive for smaller objects. The problem has become of increasing concern as decay mechanisms seem to have accelerated along with urban activity - primarily industrialisation and the burning of fossil fuels.

Water is the main agent of most stone decay mechanisms. Deterioration can be slowed if the access of water into pores is limited. Traditionally, wax and resinous coatings were applied to protect exposed stone surfaces and make them water repellent. Alkali silicates and organic polymers were later developed to strengthen and protect deteriorated stone. The function of these so called consolidants is to fortify weakened stone by permeating the pores of the stone and polymerising or curing (as for epoxy resins and alkoxysilanes) in situ. The ideal consolidant is strong, makes the surface of the stone water-repellent and protects the surface from atmospheric pollutants.

Alkoxysilanes are compounds currently under investigation at the Institute of Archaeology, and are of ten used to conserve stone, adobe, and ceramic. These compounds consist of individual silicon atoms that are attached to varying functional groups either directly or via an oxygen linkage and are called silane monomers. The silane monomers can be mixed with water and alcohol to make a non-viscous consolidant. The silane consolidant is then applied to the surface by brush or spray, and is absorbed into the pore structure by capillary action. The solution then begins to gel. The resulting polymer has a silicon-oxygen backbone that is the precursor to a type of low-temperature glass (Brinker and Scherer 1990) - i.e., an amorphous, strong, stable product. The functional groups of the silane monomer may be modified to contain non-removable alkyl groups that can impart hydrophobic qualities to the end product.

What is so appealing about the alkoxysilanes, in theory, is that during the polymerisation process they have the ability to incorporate any surface moisture present, and to bond directly to the surface hydroxyl groups of quartz. This is understandable logic for sandstones that are composed primarily of quartz, but the alkoxysilanes have also been reportedly used, with success, upon limestone and marbles. The process by which they work in these cases remains a puzzle as silanes are known to be inert towards calcium carbonate (Plueddeman 1991). If these alkoxysilanes do work upon both these types of substrates then we must 
ask - 'why'?

The actual adhesion mechanism of alkoxysilanes to reactive substrates is unknown. Also, whether or not a consolidant should have strong adhesion to its substrate is debatable. For instance, if during the polymerisation process the silane formed very strong bonds to the pore walls and then, during the drying or condensation process, the polymer shrank, stresses would develop due to the consolidant itself. These stresses could weaken the stone, thus defeating the very purpose of the treatment. Basic research into the mechanisms of alkoxysilane polymerisation, and into their long term stability, is needed, both to define the role of a stone consolidant and to optimise that consolidant's application.

Silane chemistry is complicated and both the hydrolysis and condensation reactions necessary to form the polymer are sensitive to acid or base catalysis. Depending upon the $\mathrm{pH}$ of its solution, and the encountered environment, the silane will follow different reaction mechanisms resulting in final polymers of radically differing structural properties. The aim is to define the environment encountered by the silane consolidant in situ and deduce its effect upon the alkoxysilane polymerization process.

The first step must be todetermine the $\mathrm{pH}$ of limestone, marble and sandstone surfaces. The use of indicators and neutralisation type reactions to accomplish this is well documented in the field of solid acid/base catalysts (Tanabe 1970). The data from these sources cannot be used however, because, in industry, all of the environmental parameters may be controlled and optimised; for example, a solid catalyst may be heated in order to drive off impurities and atmospheric moisture. This is not possible for a large monument and can be dangerous to weakened, decayed smaller objects.

The indicator method of acid/base surface characterization is an easy means of obtaining qualitative information on the surface $\mathrm{pH}$ of a solid. Quartz and calcite surfaces, exposed to moisture, are known to possess disordered layers that overlie the regular crystal lattice. In order to study the nature of the layer itself, it is necessary to avoid dissolution of the surface. Therefore, the indicator method is based on adsorbing the indicator directly to the solid surface by suspending the sample in a non-polar solvent. This study focuses on a preliminary investigation of the acid/base nature of some building stones in order to determine their effect upon the polymerisation processes of an alkoxysilane system.

\section{Theory}

\section{Neutralisation reactions}

The purpose of an aqueous neutralisation titration is to determine, by the slow addition of acid/base, the equivalence point; that is, the point at which the amount of acid in solution is chemically equivalent to the amount of base. Acid-base or neutralisation indicators change colour according to the concentration of the hydrogen ion in the solution. These indicators change colour over a small range of $\mathrm{pH}$ known as the colour-change interval. Each indicator will undergo a colour transition within a different $\mathrm{pH}$ range, so it is possible to chose an indicator that 
will change at a pH close to the equivalence point of the titrand.

The equilibrium between the acidic $\left(\operatorname{In}_{A}\right)$ form of the indicator and the basic (InB) form may be expressed by the equation

$$
\operatorname{In}_{A} \leftrightarrow \mathrm{H}^{+}+\operatorname{In}_{B}
$$

The colour of the indicator is determined by the ratio of the concentration of the acidic and basic forms of the indicator in the solution. Both forms of the indicator are present at any hydrogen ion concentration but the colour is not discemible to the human eye. The colour change that is visible occurs when, for the acidic colour, the ratio of [ $\operatorname{In}_{A}$ ] to [ $\left[\mathrm{In}_{B}\right.$ ] is greater than ten and vice versa for the basic colour. The colour change is gradual and occurs over approximately two $\mathrm{pH}$ units. The point at which the ratio of the concentrations of the acid and basic forms equals one is known as the middle tint.

\section{Determination of the acidic properties of a solid surface}

In order to define and measure relative surface acid strengths, the principles behind aqueous neutralisation titrations have been applied to powdered solids. The method has been adapted by researchers in the field of solid acid catalysts (Tanabe 197; Walling 1950). The resulting procedure consists of two significant steps. Firstly, the indicator must be introduced to the acid sites of the solid. Next, these sites may be titrated to determine their strengths.

The acid strength of a solid is considered to be the ability of the surface to convertan adsorbed neutral base into its conjugate acid. If the reaction proceeds via proton transfer from the surface to the adsorbate, the Hammett acidity function $\mathrm{H}_{\mathrm{O}}$ is used to express the acid strength

$$
\begin{gathered}
\mathrm{H}_{\mathrm{O}}=-\log \mathrm{a}_{\mathrm{H}+\mathrm{fB}_{\mathrm{B}} / \mathrm{f}_{\mathrm{BH}}{ }^{+}} \\
\text {or } \\
\mathrm{H}_{\mathrm{O}}=\mathrm{pk}_{\mathrm{a}}+\log [\mathrm{B}] /\left[\mathrm{BH}^{+}\right]
\end{gathered}
$$

where $\mathrm{a}_{\mathrm{H}_{+}}$refers to the proton activity; $[\mathrm{B}]$ and $\left[\mathrm{BH}^{+}\right]$refer to the concentration of the neutral base and its conjugate acid respectively; and where $f_{\mathrm{B}}$ and $f_{\mathrm{BH}}+$ refer to the corresponding activity coefficients.

If, however, the reaction proceeds via an electron pair transfer from the adsorbate to the surface then

$$
\begin{gathered}
H_{O}=-\log a_{A} f_{B} / f_{A B} \\
\text { or } \\
H_{O}=p k_{a}+\log [B] /[A B]
\end{gathered}
$$

where a refers to the activity of the Lewis acid (the electron pair acceptor).

The first step, a semi-quantitative method of determining the acidic or basic nature of the solid surface, is to add a suitable basic indicator (see Table 1) to powdered samples suspended in non-polar solvents (Tanabe 1970; Walling 1950). The indicator adsorbs onto the surface and changes either into its acid or base colour, if it changes at all. This colour change gives a rough measure of the nature of the substrate. If the indicator changes to its acidic colour, then the value 
of the $\mathrm{H}_{\mathrm{O}}$ of the surface is equal to or lower than the $\mathrm{pk}_{\mathrm{a}}$ of the indicator. Lower values of $\mathrm{H}_{\mathrm{O}}$ correspond to greater acidity values.

\begin{tabular}{|lccc|}
\hline Indicators & Acid-form colour & Base-form colour & pk \\
\cline { 2 - 3 } & yellow & red & +6.8 \\
Neutral red & yellow & red & +4.8 \\
Methyl red & yellow & red & +4.0 \\
phenylazonaphthyl amine & yellow & red & +3.3 \\
2-Dimethylaminoazo benzene & yellow & red & +2.0 \\
Benzeneazodiphenyl amine & yellow & purple & +1.5 \\
4-Dimethylaminoazo-1-nephthalene & yellow & red & +1.2 \\
Crystal violet & blue & yellow & +0.8 \\
p-Nitrobenzeneazo-(p'-nitro)diphenylamine & orange & purple & +0.43 \\
Dicinnamalacetone & yellow & red & -3.0 \\
Benzalacetophone & colourless & yellow' & -5.6 \\
Anthroquinone & colourless & yellow & -8.2 \\
\hline
\end{tabular}

Table 1. Basic indicators used for the measurement of acid strength (after Tanabe 1970)

Once the basic indicator has been adsorbed onto the solid, the suspension may be titrated with a strong base to determine the relative acid strength of the surface (Benesi 1957; Johnson 1955; Tanabe 1970; Voltz et al. 1960). The indicator binds to the acid sites of the surface and it is the $\mathrm{pK}_{\mathrm{a}}$ of the indicator that determines the level of the acid strength of the acid sites that are titrated (Johnson 1955). The indicator base is displaced by the stronger titrating base and the colour disappears or changes. Typically, amines, such as n-butylamine or benzylamine, are used for solid surface titrations. The reactions involved in the two-step indicator/titration method may be pictured as follows:

\section{1.) Addition of Indicator}
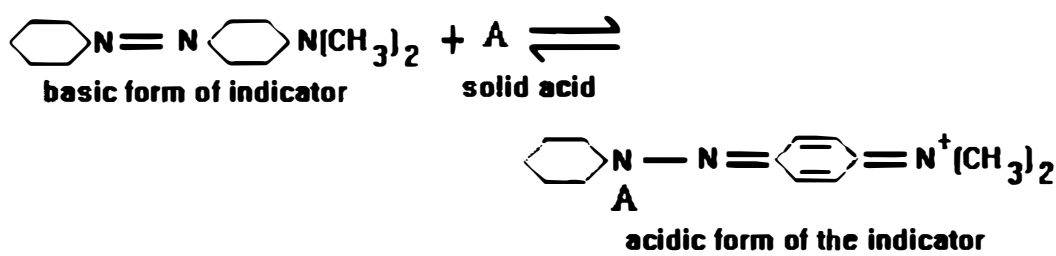

\section{2.] Amine Titration}

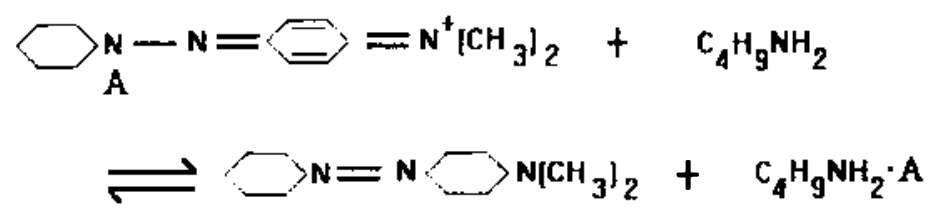


Determination of the basic properties of a solid surface

The methods of describing basic properties of solids are similar to those used in describing acidic properties. That is, the basic strength of a solid surface refers to its ability to convert an adsorbed electrically neutral acid to its conjugate, or the ability of the surface to donate an electron pair to an adsorbed acid (Tanabe 1970).

The indicator method for base surface characterization works by using an electrically neutral acid indicator (see Table 2 ) which is adsorbed onto the basic sites of the solid from a non-polar solution. If the solid has the necessary basic strength, it imparts electron pairs to the acidic indicator and changes colour to that of its conjugate base.

$$
\begin{gathered}
\mathrm{AH}+\mathrm{B} \leftrightarrow \mathrm{A}^{-}+\mathrm{BH}^{+} \\
\mathrm{H}_{\mathrm{O}}=\mathrm{pK}_{\mathrm{a}}+\log \left[\mathrm{A}^{-}\right] /[\mathrm{AH}]
\end{gathered}
$$

where $\left[\mathrm{A}^{-}\right]$is the concentration of the basic form and $[\mathrm{AH}]$ is the concentration of the acidic form of the indicator (Tanabe 1970).

$\underline{\text { Indicators }}$

Bromothymol blue

2,4,6-Trinitroniline

2,4-Dinitroaniline

4Chloro-2-nitroaniline

4-Nitroaniline

4-Chloroaniline

\section{Acid-fonn colour}

yellow

yellow

yellow

yellow

yellow

yellow
Base-form colour

$\begin{array}{cr}\text { green } & 7.2 \\ \text { reddish-orange } & 12.2 \\ \text { violet } & 15.0 \\ \text { orange } & 17.2 \\ \text { orange } & 18.4 \\ \text { pink } & 26.5\end{array}$

Table 2. Acidic indicators used forthemeasurement of basic strength (after Tanabe 1970)

The approximate value of the basic strength on the surface is taken to be equal to the $\mathrm{pK}_{\mathrm{a}}$ of the adsorbed indicator at which the intermediate colour appears.

\section{Experimental techniques}

\section{Indicator method materials and procedure}

Four types of stone samples (sandstone, weathered sandstone, marble and limestone) were ground and sieved through 500 micron mesh. Then $0.1 \mathrm{~g}$ of a powdered sample was placed in a capped bottle. $2 \mathrm{ml}$ of cyclohexane containing $0.2 \mathrm{mg}$ of the indicator, either methyl red or bromothymol blue, was added. The sample was briefly shaken and the colour change noted.

\section{Preparation of samples for the comparison of pre-treatment}

In order to compare the effect of different pre-treatments, five sets of samples were prepared. Two were kept at a constant relative humidity of 55\%. The other three sample sets were heated at $80^{\circ} \mathrm{C}$ for 14 hours. The sample sets were then removed and placed directly into different environments to equilibrate, (ambient 
30-40 \% R.H., 50-60\% R.H. and 10-15\% R.H.) for 24 hours.

\section{Preparation of stone-silane-water-ethanol systems}

Unheated samples stored at $55 \%$ relative humidity were stirred with $20 \mathrm{ml}$ of a 4:1:3 molar ratio solution of water:tetraethoxysilane (TEOS):ethanol. These solutions were placed in a number of test tubes and a series of indicators were used to determine the $\mathrm{pH}$ of the solutions. The colours of the stone-silane-water solution and indicator were then compared to that of the control (Water:TEOS:Ethanol with no stone).

\section{Interpretation and results}

The history of the sample may have some effect upon the acid/base character of the solid surface. The method of preparation, for instance, is known to have a great effect upon the nature of the surface. Washing with distilled water or other solvents may wash away impurities, while heating may increase the acidic nature of the substrate or, in some cases, the basicity (Tanabe 1970).

All samples turned the acid form of bromothymol blue, indicating that they have an $\mathrm{H}_{\mathrm{O}}$ lower than 7.1. For the sandstones and quartz this came as no surprise; however, the indication of an acidic carbonate surface was unexpected, as carbonates give basic aqueous solutions. The samples were then mixed with the methyl red indicator. The samples all turned red, the acidic form of the indicator, which means that the $\mathrm{H}_{\mathrm{O}}$ is less than or equal to 4.8. The marble sample turned a light pink, with some slight evidence of yellow areas (see Table 3).

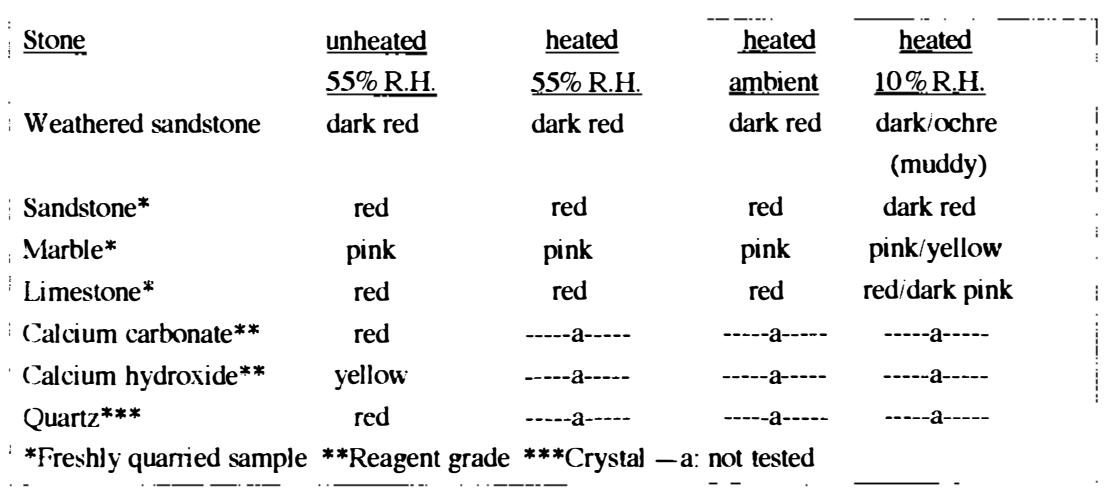

Table 3. Methyl red adsorption and colour change on powdered samples

These results reflect the acid/base characterisation of the samples in a nonpolar solvent. However, alkoxysilanes are applied to the stones in water-alcohol solutions. The surface species, cement components, and salts that may be present upon or within the stone samples are water soluble thus presenting a problem. Since the aim of the study is to look at the surface characterisation of stone with regard to its influence upon the alkoxy-silane polymerisation process, the decision was made to look at stone-silane-water-ethanol systems. 
The $\mathrm{pH}$ of the control solution (water:TEOS:ethanol and no stone) was approximately 5-6. Please note that the silane solution formed two phases. The lower phase, in contact with the stone was the phase studied as it gelled in contact with the stone. Both phases were analysed with Fourier Transform Infrared Spectroscopy and found to contain silane as well as water and ethanol. The pH of the solution appeared to increase in the presence of limestone and marble to approximately 7-8 or more, the solutions in contact with the sandstone and weathered sandstone remained the same. The silane solution was then drained from the samples, and the stone samples were washed with distilled water. The samples were allowed to dry for 18 hours at ambient relative humidity. $2 \mathrm{ml}$ of cyclohexane containing the indicator methyl red were then added to each sample and the solvent was allowed to evaporate off. The 'stained' stone (stone+methyl red only - no pre-treatments) samples were compared with stained silane-treated stone samples under reflectance optical microscopy. Both of these sample sets were then compared with stone that was allowed to sit in a 4:3 molar ratioof water to ethanol for $\mathbf{2 4}$ hours (see Table 4).

\section{Oplical microscopy of melhyl red stained samples}

The stained stone powders were obseried under low magnification via optical microscopy. Colour photographs of the powders were made, but for publishing purposes, the results will be given here as comments in a table format. Please note that the control sample set of stone-water-ethanol gave results intermediate between that of the untreated and treated samples (see Table 4).

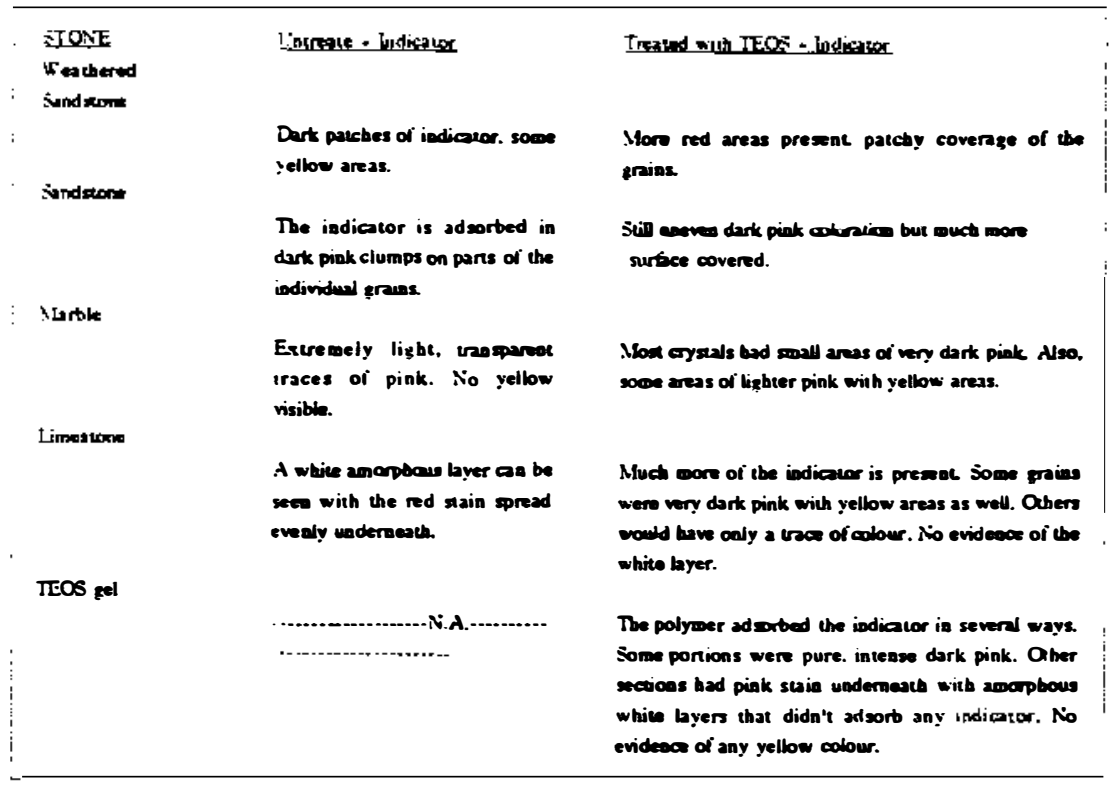

Table 4 Colour change of treated and control samples 


\section{Discussion}

Research on solid substrates reported in the industry literature, primarily regarding the nature of these substrates as to their catalytic perfonnance, has shown that silicon oxides and calcium carbonate have both acidic and basic properties (Tanabe 1970).

In the determination of the acidic or basic nature of a solid substrate the degree of hydration is an important factor to consider. Quartz is generally thought to be 'unweatherable' but it is slightly soluble and Alexanian (in Iler 1979) found that quartz had a surface layer of amorphous silica approximately $100 \AA$ thick that could be removed with HF but reformed in ambient humidity. One possible model for a quartz surface may be pictured as below

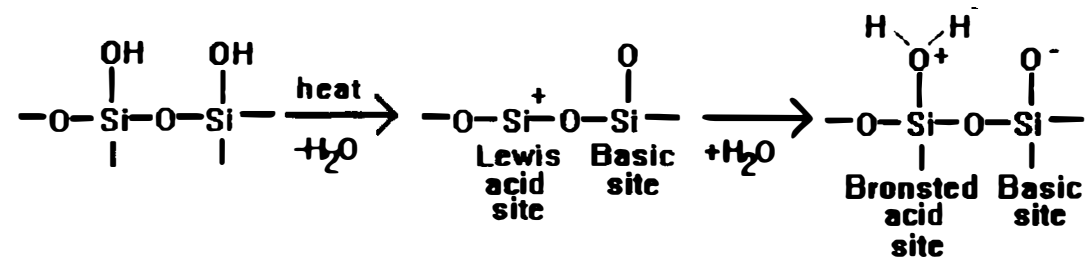

However, the Si-O-Si bonds at the surface may also be split by hydrolysis when in contact with water. Developing a model for a calcite-water interface is much more difficult than for a quartz-water interface due to the high reactivity and mobility of the surface ions. Even in a closed system (no $\mathrm{CO}_{2}$ ) one monolayer of the surface is exchanged with the aqueous medium within one day (Cicerone et al. 1992).

Carbonates are mainly weathered by dissolution governed by the following net equation:

$$
\mathrm{CaCO}_{3}+\mathrm{H}_{2} \mathrm{CO}_{3} \leftrightarrow \mathrm{Ca}^{2+}+2 \mathrm{HCO}_{3}^{-}
$$

The dissolution process occurs instages. Threepossible steps are(afterOllier 1979)

1) The dissolution of calcite at the solid-liquid interfaceintoCa ${ }^{2+}$ and $_{3} \mathrm{CO}_{3}{ }^{2-}$ ions.

2) The reactions between $\mathrm{H}_{2} \mathrm{CO}_{3}, \mathrm{CO}_{3}^{2-}$ to give $\mathrm{HCO}_{3}$ -

a) $\mathrm{CO}_{2(\mathrm{aq})}+\mathrm{H}_{2} \mathrm{O} \leftrightarrow \mathrm{HCO}_{3}^{-}+\mathrm{OH}^{-}$

b) $\mathrm{H}_{2} \mathrm{CO}_{3} \leftrightarrow \mathrm{H}^{+}+\mathrm{HCO}_{3}^{-}$

c) $\mathrm{H}^{+}+\mathrm{CO}_{3}^{2-} \leftrightarrow \mathrm{HCO}_{3}^{-}$

3) If the solution is in contact with $\mathrm{CO}_{2}(\mathrm{~g})$ the $\mathrm{CO}_{2}(\mathrm{~g})$ may dissolve and contribute $\mathrm{CO}_{2(\mathrm{aq})}$ to the solution thus increasing dissolution (step 2a):

$$
\mathrm{CO}_{2(\mathrm{~g})}+\mathrm{H}_{2} \mathrm{O} \leftrightarrow \mathrm{CO}_{2(\mathrm{aq})}
$$

The problem with these equations is that they present the dissolution steps as if the solution was in contact with a perfectly regular crystal surface. This is actually not the case. The presence of protonated surface carbonate and of hydroxide-water associated with surface calcium ions has been determined by XPS (Cicerone et al. 1992). This had, in the past, been interpreted as being due 
to the formation of surface complexes on an inert basic array of lattice ions, as in the equations above. However, the latest work has developed surface hydration models which propose that a strongly hydrated and disordered calcium carbonate layer overlies the calcite crystals (Cicerone et al. 1992). While the main surface sites are thought to be, originally, $\mathrm{Ca}^{2}+$ and $\mathrm{CO}_{3}{ }^{-}$it is thought that the presence of other species, (i.e., $\mathrm{HCO}_{3}^{-}, \mathrm{CaOH}^{+}, \mathrm{CaCO}_{3} \mathrm{H}, \mathrm{CO}_{3} \mathrm{H}, \mathrm{CO}_{3} \mathrm{Ca}^{+}$, $\mathrm{CaOH}_{2}{ }^{+}, \mathrm{CaOH}, \mathrm{CaO}^{-}$and $\mathrm{CaCO}_{3}$ ) could be due to adsorption of the species onto the hydrated surface from solution.

Overall, both quartz and calcite crystals exposed to an open environment would possess hydrated, disordered surface layers. This is an important point when considering the effectiveness of alkoxysilanes as stone consolidants. Their desirability is based upon their ability to bond directly to the hydroxyl ions upon the crystal surface. If the surface is inconsistent then the effectiveness of this bonding may be reduced. This fact is born out by literature within the fields of materials science and engineering which recommend cleaned and heat-treated surfaces for maximum increases in the tensile strength of composite materials (Plueddeman 1991).

Both the hydrolysis and condensation reactions are greatly affected by the presence of acids or bases which will change the structure of the end alkoxysilane polymer. Many alkoxysilane treatments are applied as aqueous solutions which, unlike the non-polar solvents used to determine surface acidity, may dissolve the surface of the rock grains/crystals. The overall $\mathrm{pH}$ of the solution will determine the final polymer structure but what affect does it have upon the bonding mechanism? All of the stones tested in this experiment showed an increase in surface acidity when treated with the ethanol-water solution and an even greater increase when treated with the silane solution. The crystals do not show an even increase over the surface but patches of greater intensity varying from grain to grain. The increase in surface acidity with the ethanol-water treatment may be due to protonation of surface sites by species formed from the dissolution processes. The increase in acidity with the TEOS treatment may be due to adsorption of the silane onto the surface, but more work must be done to determine this conclusively.

\section{Conclusion}

Through the use of acid-base indicators, this study has concluded that both the sandstones and the limestones studied have acidic surfaces in non-aqueous, nonpolar solvents. However, perhaps more important is the variability of these surfaces which appears to depend upon a variety of factors. The weathered sandstone was the most susceptible to environmental changes as far as surface acidity is concerned (see Table 3 ) and this raises problems associated with stone conservation/consolidation. The stone that needs consolidation is weathered and will have been exposed to different pollutants, salts or previous conservation treatments. How does this difference in environment affect alkoxysilane polymerisation? 
The nature of the solvent-polymer/monomer-stone interaction is extremely important in the performance of the final polymer as a consolidant. Alkoxysilanes are often applied 'neat' - that is, without water and a solvent or as aqueous solutions. These different solvent environments will encounter, perhaps, different surface conditions. How will this affect the life and effectiveness of the consolidant? More work must be done towards a coherent strategy to define the parameters that will contribute to successful consolidation.

\section{Acknowledgements}

I would like to thank Professor David Williams at the Chemistry Department, UCL for his valuable help and great ideas. Special thanks to Dr. Ziad Al Ahmed for his encouragement at the beginning of this project. Last, but not least, I would like to thank my supervisors, Dr. Dafydd Griffiths and Dr. Clifford Price, both at the Institute of Archaeology, UCL, for their support.

\section{References}

Benesi, H. A. 1957. Acidity of catalyst surfaces II. Amine titration using Hammett indicators. Journal of Physical Chemistry, 61: 970-3.

Brinker, C. J. and Scherer, G. W. 1990. Sol-Gel Science. New York: Academic Press.

Cicerone, D., Regazzoni, A. E. and Blessa, M. A. 1992. Electrokinetic properties of the calcite/water interface in the presence of magnesium and organic matter. Journal of Colloid and Interface Science, 154: 423-33.

Iler, R. 1979. The Chemistry of Silica. Chichester: John Wiley and Sons.

Johnson, O. 1955. Acidity and polymerization activity of solid acid catalysts. Journal of Physical Chemistry, 59: 827-31.

Ollier, C. 1984. Weathering. London: Longman.

Plueddeman, E. P. 1991. Silane Coupling Agents. London: Plenum Press.

Tanabe, K. 1970. Solid Acids and Bases. London: Academic Press.

Voltz, S. E., Hirschler, A. E. and Smith, A. 1960. Hammett acidities of chromia catalysts. Journal of Physical Chemistry, 64: 1594-6.

Walling, C. 1950. The acid strength of surfaces. Journal of the American Chemical Society, 72: 1164-8. 